\title{
DISCOVERY OF KILOHERTZ QUASI-PERIODIC OSCILLATIONS IN GX 17+2
}

\author{
Rudy Wijnands, ${ }^{1}$ Jeroen Homan, ${ }^{1}$ Michiel van der Klis, ${ }^{1}$ Mariano Méndez, ${ }^{1,2}$ Erik KuUlkers, ${ }^{3}$ Jan van Paradijs, ${ }^{1,4}$ \\ Walter H. G. Lewin, ${ }^{5}$ Frederick K. Lamb, ${ }^{6}$ Dimitrios Psaltis, ${ }^{6}$ and Brian Vaughan ${ }^{7}$ \\ Received 1997 August 15; accepted 1997 October 1; published 1997 October 30
}

\begin{abstract}
We observed the low-mass X-ray binary and $Z$ source GX $17+2$ with the Rossi X-Ray Timing Explorer during 1997 February 6-8, April $1-4$, and July 26-27. The X-ray color-color diagram shows a clear $Z$ track. Two simultaneous $\mathrm{kHz}$ quasi-periodic oscillations (QPOs) are present in each observation, whose frequencies are well correlated with the position of the source on the $Z$ track. At the left end of the horizontal branch (HB), only the higher frequency peak is observed, at $645 \pm 9 \mathrm{~Hz}$, with an rms amplitude of $5.7 \% \pm 0.5 \%$ and an FWHM of $183 \pm 35 \mathrm{~Hz}$. When the source moves down the $Z$ track to the upper normal branch, the frequency of the $\mathrm{kHz}$ QPO increases to $1087 \pm 12 \mathrm{~Hz}$, and the rms amplitude and FWHM decrease by a factor of 2 . Farther down the $Z$ track, the QPO becomes undetectable, with rms upper limits typically of $2.0 \%$. Halfway down the HB, a second QPO appears in the power spectra with a frequency of $480 \pm 23 \mathrm{~Hz}$. The frequency of this QPO also increases when the source moves along the $Z$ track, up to $781 \pm 11 \mathrm{~Hz}$ halfway down the normal branch, while the rms amplitude and FWHM stay approximately constant at $2.5 \%$ and $70 \mathrm{~Hz}$. The QPO frequency difference is constant at $293.5 \pm 7.5 \mathrm{~Hz}$. Simultaneously with the $\mathrm{kHz}$ QPOs, we detect HB QPOs (HBOs). The simultaneous presence of HBOs and $\mathrm{kHz}$ QPOs excludes the magnetospheric beat-frequency model as the explanation for at least one of these two phenomena.
\end{abstract}

Subject headings: accretion, accretion disks — stars: individual (GX 17+2) - stars: neutron - X-rays: stars

\section{INTRODUCTION}

GX $17+2$ is a bright low-mass $X$-ray binary and $Z$ source (Hasinger \& van der Klis 1989). Along the $Z$ track traced out in the X-ray color-color diagram (CD), the mass accretion rate $(M)$ is thought to increase from the horizontal branch (HB) via the normal branch (NB) to the flaring branch (FB). On the HB and upper NB, 18-60 Hz quasi-periodic oscillations (QPOs) are present (called HBOs), and on the lower NB and FB, 5-20 $\mathrm{Hz}$ QPOs (N/FBOs) are present. Atoll sources have no $Z$ track in the CD (Hasinger \& van der Klis 1989) and do not exhibit HBOs or N/FBOs. ${ }^{8}$ However, many show $\mathrm{kHz}$ QPOs between 300 and $1200 \mathrm{~Hz}$ (see van der Klis 1997 for a recent review). Usually, two simultaneous kHz QPOs (hereafter "twin peaks") are seen, whose frequencies increase with $\dot{M}$ while their difference (the twin peak separation) remains constant. In X-ray bursts oscillations are seen with a frequency once or twice the twin peak separation. This strongly suggests a model in which the burst oscillation frequency is the spin frequency (or twice

\footnotetext{
'Astronomical Institute "Anton Pannekoek," University of Amsterdam, and Center for High Energy Astrophysics, Kruislaan 403, NL-1098 SJ Amsterdam, The Netherlands; rudy@astro.uva.nl, homan@astro.uva.nl, michiel@ astro.uva.nl,mariano@astro.uva.nl, jvp@astro.uva.nl.

${ }^{2}$ Facultad de Ciencias Astronómicas y Geofísicas, Universidad Nacional de La Plata, Paseo del Bosque S/N, 1900 La Plata, Argentina.

${ }^{3}$ Astrophysics, University of Oxford, Nuclear and Astrophysics Laboratory, Keble Road, Oxford OX1 3RH, England, UK; e.kuulkers1@physics. oxford.ac.uk.

${ }^{4}$ Departments of Physics, University of Alabama at Huntsville, Huntsville, AL 35899.

${ }^{5}$ Department of Physics and Center for Space Research, Massachusetts Institute of Technology, Cambridge, MA 02139; lewin@space.mit.edu.

${ }^{6}$ Departments of Physics and Astronomy, University of Illinois at UrbanaChampaign, Urbana, IL 61801; f-lamb@uiuc.edu, demetris@astro.uiuc.edu.

${ }^{7}$ Space Radiation Laboratory, California Institute of Technology, 220-47 Downs, Pasadena, CA 91125; brian@thor.srl.caltech.edu.

${ }^{8}$ Although in several of them, broad QPO-like peaks are found with frequencies around $20 \mathrm{~Hz}$ (e.g., Hasinger \& van der Klis 1989; Wijnands \& van der Klis 1997).
}

that), the higher $\mathrm{kHz}$ QPO frequency is some inner disk frequency, and the lower $\mathrm{kHz}$ QPO frequency is their beat.

So far, two $Z$ sources have shown $\mathrm{kHz}$ QPOs: Sco X-1, with frequencies in the range $550-1100 \mathrm{~Hz}$ (van der Klis et al. 1996a, 1997b), and GX 5-1 (325-896 Hz; van der Klis et al. 1996b). Both sources showed twin peaks. In GX 5-1 the QPO frequencies increased from the left end of the HB to the upper NB (van der Klis et al. 1996b), and they increased in Sco X-1 from the upper NB to the FB (van der Klis et al. 1996a, $1997 \mathrm{~b}$ ). In view of the similarities between the $\mathrm{kHz}$ QPOs in $Z$ and atoll sources, both are most likely caused by the same mechanism. The only difference so far is that in atoll sources the peak separation is constant, whereas in Sco X-1 it decreases when the QPO frequencies increase. In this Letter, we report the discovery of twin $\mathrm{kHz}$ QPOs in the $Z$ source GX $17+2$ (see also van der Klis et al. 1997a).

\section{OBSERVATIONS AND ANALYSIS}

We observed GX 17+2 with the PCA on board the Rossi X-Ray Timing Explorer on 1997 February 6-8, April 1-4, and July 26-27. A total of $120.5 \mathrm{ks}$ of good data were obtained. During February $8(1.1 \mathrm{ks})$ and a small part of February 7 (7.8 ks) and July $27(4.8 \mathrm{ks})$, only four of the five detectors were on. The source then covered parts of the $Z$ track that were also well covered with five detectors. As adding the four-detector data did not improve the results significantly, we used only the five-detector data to have the most accurate $\mathrm{CD}$ possible. Data were collected with $16 \mathrm{~s}$ time resolution in 129 photon energy bands (energy range, $2-60 \mathrm{keV}$ ) and simultaneously with $122 \mu$ s time resolution in four bands: $2-5,5-6.4$, 6.4-8.6, and 8.6-60 keV.

We made power density spectra from the $122 \mu$ s data, using $16 \mathrm{~s}$ data segments. To measure the $\mathrm{kHz}$ QPOs we fitted the 96-4096 Hz power spectra with a function described by a constant, one or two Lorentzian peaks, and a broad sinusoid to represent the dead-time modified Poisson noise (Zhang et al. 1995; Zhang 1995). The Very Large Event window (van 


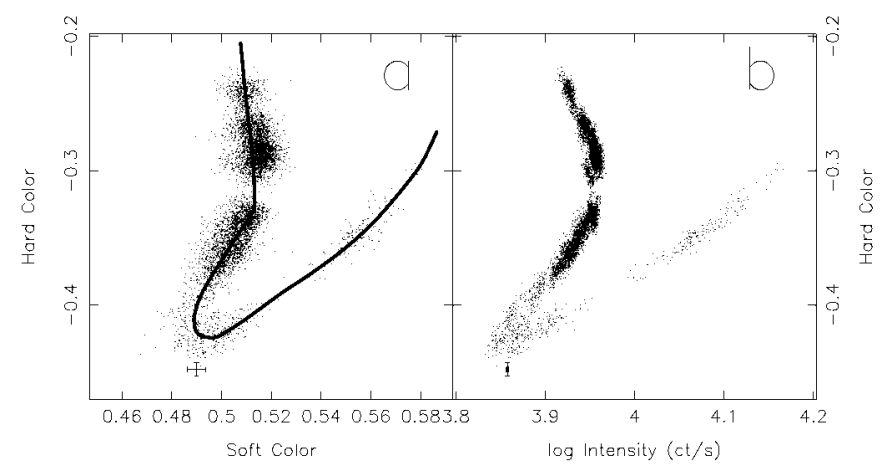

FIG. 1.-Color-color diagram $(a)$ and hardness-intensity diagram $(b)$ of GX $17+2$. The soft color is the logarithm of the ratio of the count rates between 3.5-6.4 and 2.0-3.5 keV; the hard color is the logarithm of the ratio of the count rates between $9.7-16.0$ and $6.4-9.7 \mathrm{keV}$; and the intensity is the logarithm of the count rate in the photon energy range $2.0-16.0 \mathrm{keV}$. The background, which is almost negligible (50 counts $\mathrm{s}^{-1}$ in the energy range $2.0-16.0 \mathrm{keV}$ ), was subtracted, but the intensity was not dead-time corrected. All points are $16 \mathrm{~s}$ averages. The heavy line in the $\mathrm{CD}$ is the spline that was used to determine $S_{z}$. Typical error bars are shown at one of the points.

der Klis et al. 1997b) was set to $55 \mu \mathrm{s}$, so that its effect on the Poisson noise was small enough that it could be absorbed by the broad sinusoid. To measure the HBOs we fitted the 8-256 Hz power spectra with a constant, one or two Lorentzian peaks, and a power law representing the continuum. Differential dead time (van der Klis 1989) was negligible. We determined errors using $\Delta \chi^{2}=1.0$ and upper limits using $\Delta \chi^{2}=2.71$, corresponding to a $95 \%$ confidence level. Upper limits on the $\mathrm{kHz}$ QPOs were determined using a fixed FWHM $(100 \mathrm{~Hz})$. In the presence of one $\mathrm{kHz}$ peak, the upper limit on the other was determined by fixing its frequency at the frequency of the detected QPO plus or minus the mean peak separation (depending on whether the lower or higher frequency QPO was detected, respectively). Upper limits on the HBO second harmonic were determined using a fixed FWHM $(10 \mathrm{~Hz})$ and a frequency twice that of the first harmonic.

In the $\mathrm{CD}$ and the hardness-intensity diagram (HID) we used for the soft color the log of the 3.5-6.4/2.0-3.5 keV count rate ratio, for the hard color the $\log$ of the 9.7-16.0/6.4-9.7 keV ratio, and as intensity the log of the count rates in the 2.0-16.0 $\mathrm{keV}$ band.

We used the $S_{z}$ parameterization (Wijnands et al. 1997b and references therein) for measuring the position along the $Z$ track. The HB/NB vertex is at $S_{z}=1.0$, and the NB/FB vertex is at $S_{z}=2.0$. By using logarithmic values for the colors, $S_{z}$ does not depend on the values of the colors but only on their variations (Wijnands et al. 1997b). We selected the power spectra according to $S_{z}$ and determined the average $S_{z}$ value for each average power spectrum. The $S_{z}$ error bars represent the standard deviation of the $S_{z}$ distributions.

\section{RESULTS}

In April the source traced out a full $Z$ track in the $C D$ and HID (Fig. 1). In February the HB/NB vertex and the upper NB were covered, and in July the HB was covered. The February data fall right on top of the April data, indicating that the $Z$ track did not move appreciably between February and April. The July data have higher count rate and higher soft color. Such shifts have been observed before in GX 17+2 (Kuulkers et al. 1997), and in other $Z$ sources (Cyg X-2: Hasinger et al. 1990; GX 5-1: Kuulkers et al. 1994). In all observations we detected

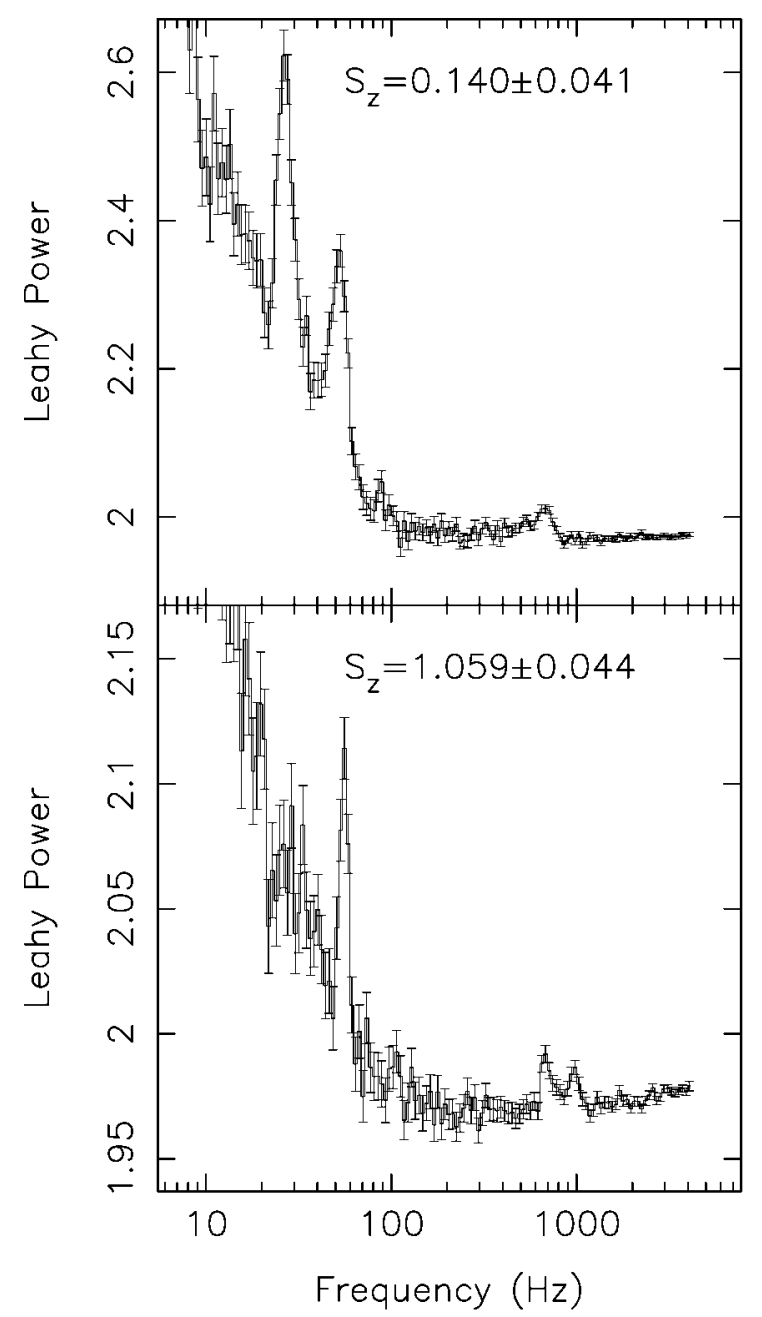

FIG. 2.-Typical Leahy normalized power spectra in the energy range 6.4-60 $\mathrm{keV}$. In the top panel, for $S_{z}=0.140 \pm 0.041$, clearly the HBO, its second harmonic, and the higher frequency QPO at $675 \mathrm{~Hz}$ are visible. In the bottom panel, for $S_{z}=1.059 \pm 0.044$, the HBO fundamental, the lower frequency QPO $(680 \mathrm{~Hz})$, and the higher frequency QPO $(986 \mathrm{~Hz})$ are visible. The upward trend of the power at $\mathrm{kHz}$ frequencies, best seen in the bottom panel, is a result of instrumental dead time.

$\mathrm{kHz}$ QPOs in the $8.6-60 \mathrm{keV}$ band. In the $6.4-8.6 \mathrm{keV}$ band, the QPOs were not, or were marginally, detected. Combining both bands, the significance of the QPOs increased, although the rms amplitude decreased a little. Therefore, we used the 6.4-60 keV range for the analysis of the QPOs.

We detected the $\mathrm{kHz}$ QPO only for $S_{z}<1.5$. Typical power spectra are shown in Figure 2. Note the simultaneous presence of the HBO and its harmonic (upper frame) and the $\mathrm{kHz}$ QPOs. The properties of the QPOs as a function of $S_{z}$ are shown in Figure 3. The higher frequency QPOs increase in frequency from $645 \pm 9 \mathrm{~Hz}$ at $S_{z}=0.07 \pm 0.05$ to $1087 \pm 12 \mathrm{~Hz}$ at $S_{z}=1.44 \pm 0.05$ (Fig. $3 a$ ), while their rms amplitude and FWHM decrease from $5.7 \% \pm 0.5 \%$ to $1.5 \% \pm 0.4 \%$ (Fig. $3 c$ ) and from $183 \pm 35 \mathrm{~Hz}$ to $21 \pm 20 \mathrm{~Hz}$. Above $S_{z}=1.5$, the higher frequency QPO is undetectable (upper limits typically of $2.5 \% \mathrm{rms}$ ). For $S_{z}<0.5$ the lower frequency QPO is not detected, with upper limits near $2.5 \% \mathrm{rms}$. From $S_{z}=0.56$ \pm 0.04 to $S_{z}=1.34 \pm 0.05$, the lower frequency QPO is detected at a frequency increasing from $480 \pm 23$ to $781 \pm$ $11 \mathrm{~Hz}$ (Fig. 3a). The rms amplitude (Fig. 3e) and FWHM of the lower frequency QPO are about constant at $2 \%-3 \%$ and 


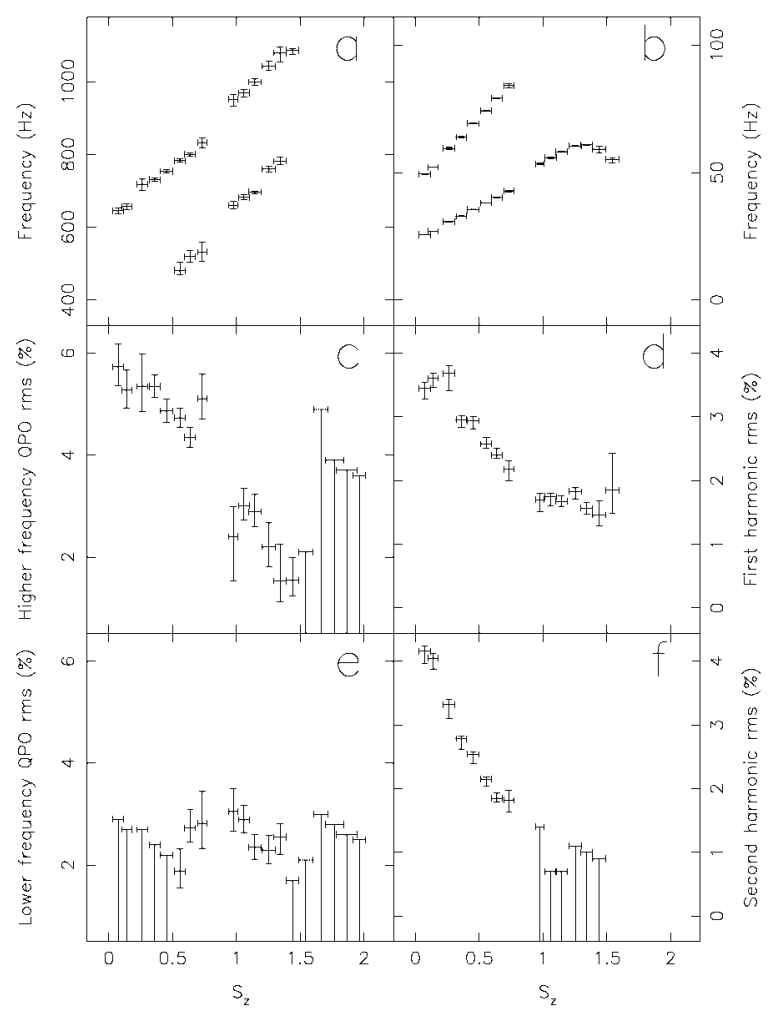

FIG. 3.- (a) Frequency of the kHz QPOs; $(b)$ frequency of the first and second harmonic of the HBO; $(c)$ the rms amplitude of the higher frequency $\mathrm{kHz} \mathrm{QPO} ;(d)$ the rms amplitude of the first harmonic of the HBO; $(e)$ the rms amplitude of the lower frequency $\mathrm{kHz} \mathrm{QPO}$; and $(f)$ the rms amplitude of the second harmonic of the HBO as a function of $S_{z}$.

$50-150 \mathrm{~Hz}$. When both QPOs are detected, the frequency difference is consistent with being constant at $293.5 \pm 7.5 \mathrm{~Hz}$.

The QPOs were strongest at high energy. We combined all data with $S_{z}<0.5\left(S_{z}=0.23 \pm 0.14\right)$ and with $S_{z}$ between 1.0 and $1.3\left(S_{z}=1.15 \pm 0.10\right)$ to detect the QPOs at lower energy. When $S_{z}>1.0$, both QPOs are seen. Both get stronger with energy, and both have about the same amplitude in each band (see Table 1). When $S_{z}<0.5$, only the higher frequency QPO is seen. Apart from an overall increase in amplitude, its energy dependence is approximately the same as for $S_{z}>1.0$ (see Table 1).

The $\mathrm{kHz}$ QPOs and HBO and its second harmonic occur simultaneously (see Fig. 2). The HBO frequency increases from the left end of the HB onto the upper NB (Fig. $3 b$ ). At $S_{z}=$ $1.34 \pm 0.05$, it reaches a maximum of $61 \pm 0.2 \mathrm{~Hz}$, and farther down the NB it decreases again. On the HB the rms amplitude of the fundamental decreases with $S_{z}$; on the NB it is about constant at $1.5 \%-2 \%$ (Fig. $3 d$ ). Above $S_{z}=1.6$, it is not detected with upper limits of $2.0 \% \mathrm{rms}$. The rms amplitude of the second harmonic decreases on the HB with $S_{z}$ (Fig. $3 f$ ). A detailed analysis of HBOs and N/FBOs will be reported by Homan et al. (1997).

\section{DISCUSSION}

We detected two simultaneous $\mathrm{kHz}$ QPOs in GX 17+2. After Sco X-1 (van der Klis et al 1996a, 1997b) and GX 5-1 (van der Klis et al. 1996b), this is the third $Z$ source displaying $\mathrm{kHz}$ QPOs.. The QPO frequency increases down the HB and onto

${ }^{9}$ Recently, we also found kHz QPOs in Cyg X-2 (Wijnands et al. 1997a).
TABLE 1

Kilohertz QPO rms Amplitude versus Photon ENergy ${ }^{\mathrm{a}}$

\begin{tabular}{|c|c|c|c|}
\hline \multirow[b]{2}{*}{$\begin{array}{l}\text { ENERGY } \\
(\mathrm{keV})\end{array}$} & \multirow{2}{*}{$\frac{S_{z}=0.5 \pm 0.14}{\begin{array}{c}\text { Upper Peak rms } \\
(\%)\end{array}}$} & \multicolumn{2}{|c|}{$S_{z}=1.15 \pm 0.10$} \\
\hline & & $\begin{array}{c}\text { Upper Peak rms } \\
(\%)\end{array}$ & $\begin{array}{c}\text { Lower Peak rms } \\
(\%)\end{array}$ \\
\hline $2-5.0$ & $<1.9$ & $<1.1$ & $<1.2$ \\
\hline $5.0-6.4$ & $3.4 \pm 0.5$ & $2.0 \pm 0.5$ & $<1.9$ \\
\hline $6.4-8.6$ & $3.9 \pm 0.5$ & $2.2 \pm 0.4$ & $1.7 \pm 0.5$ \\
\hline $8.6-60$. & $7.4 \pm 0.2$ & $3.6 \pm 0.3$ & $4.1 \pm 0.2$ \\
\hline $5.0-60^{\mathrm{b}}$ & $5.2 \pm 0.2$ & $2.1 \pm 0.2$ & $2.3 \pm 0.2$ \\
\hline
\end{tabular}

${ }^{\text {a }}$ All errors correspond to $\Delta \chi^{2}=1$. The upper limits correspond to the 95\% confidence level.

${ }^{\mathrm{b}}$ Added for comparison with Cyg X-2; Wijnands et al. 1997a.

the NB. For atoll sources an increase in frequency is thought to be due to an increase in $M$. Because of the very similar properties of the $\mathrm{kHz}$ QPOs in atoll sources and in GX 17+2, our observations confirm that $\dot{M}$ indeed increases when the source moves down the $Z$ track, even when (on the NB) the count rate decreases, as previously concluded by Hasinger \& van der Klis (1989).

Although the behavior of the X-ray spectra changes significantly at $S_{z}=1$ when the source enters the NB, and the Xray flux begins to decrease with $S_{z}$ there, the frequencies of all QPOs continue to increase with $S_{z}$ in the same manner as on the HB. The only evidence that the rapid variability is affected as the source enters the NB is by the HBO rms amplitude. Decreasing with $S_{z}$ on the HB, the amplitude is about constant on the NB.

Between $S_{z}=1.4$ and 1.5, the HBO frequency decreases. A similar decrease in HBO frequency on the NB for GX $17+2$ was seen in the "variable-frequency" QPO on the NB reported by Wijnands et al. (1996). Although supporting evidence for a decreasing HBO frequency on the NB was found in Ginga data on Cygnus X-2 (Wijnands et al. 1997b), it was unclear if the "variable-frequency" QPO in GX 17+2 was indeed the HBO (see Kuulkers et al. 1997). From Figure $3 b$ it is now evident that indeed it was and that it was the fundamental and not, as proposed by Kuulkers et al. (1997), the second harmonic. The data reported here and by Wijnands et al. (1996) show that the behavior of the HBO did not change significantly over $1 \mathrm{yr}$. In the data presented here, the HBO does not decrease to the same frequency as in 1996; this is due to a lack of data on the NB.

Beat-frequency models (BFMs) are the best models so far to explain the $\mathrm{kHz}$ QPOs in atoll sources. In GX $17+2 \mathrm{kHz}$, QPOs and HBOs are observed simultaneously, so if the magnetospheric BFM explains the HBO (Alpar \& Shaham 1985; Lamb et al. 1985), it cannot explain the kHz QPOs (the same is true for Sco X-1; van der Klis et al. 1997b). The sonic-point BFM for the $\mathrm{kHz}$ QPOs in atoll sources (Miller, Lamb, \& Psaltis 1997) assumes that the highest frequency is due to orbital motion around the neutron star at the sonic radius and the lower frequency to its beat with the neutron star spin frequency. This model predicts a constant twin peak separation, as observed for atoll sources. According to this model, the spin period of the neutron star in GX $17+2$ would be $3.41 \pm 0.03 \mathrm{~ms}$.

In Sco X-1 the twin peak separation decreases when the frequencies of the $\mathrm{kHz}$ QPOs increase (van der Klis et al. 1997b). Although the peak separation in GX 17+2 is consistent with being constant, its error bars are larger than in Sco X-1, and a decrease similar to that in Sco X-1 cannot be excluded (see Fig. 4). Note that the decrease of the peak separation in Sco X-1 was observed when this source was moving down the 


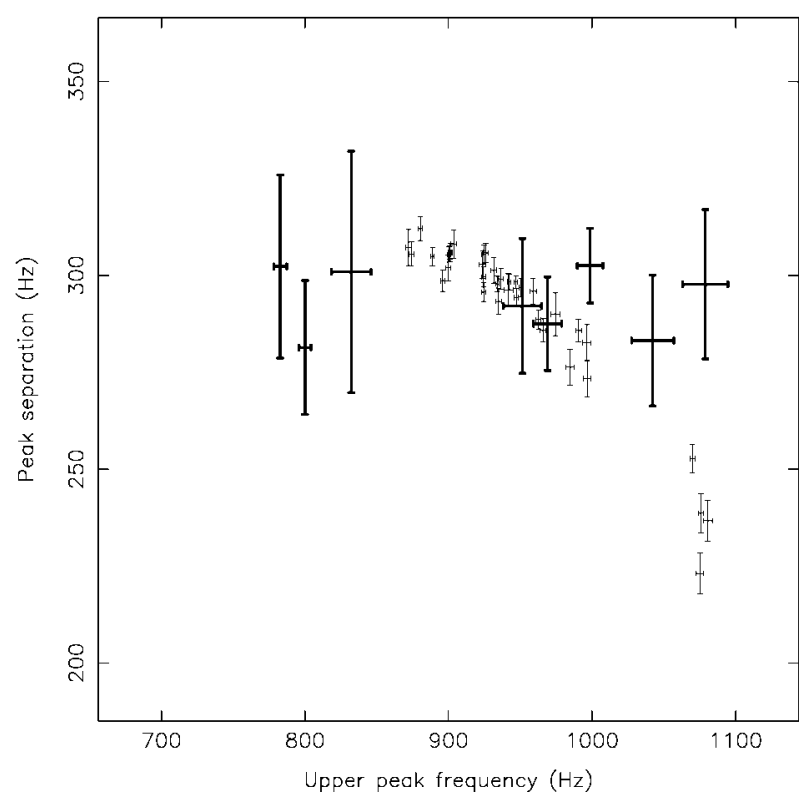

FIG. 4.-The kHz peak separation vs. the frequency of the higher frequency QPO. The heavy points with the large error bars are for GX $17+2$, and the light points with the small error bars are for Sco X-1 (taken from van der Klis et al. 1997b, their Fig. 3a).

NB onto the beginning of the FB. We could not observe the $\mathrm{kHz}$ QPOs this far down the $Z$ track in GX $17+2$.

Another explanation that has been proposed for $\mathrm{kHz}$ QPOs is the photon bubble oscillation (PBO) model of Klein et al. (1996). These oscillations have been modeled to occur below $0.4 L_{\text {Edd }}$ and only weakly (or not at all) above $1.1 L_{\text {Edd }}$ (Klein et al. 1996; R. Klein 1997, private communication). The amplitudes of the PBOs decrease and their frequencies increase with $\dot{M}$, just as we observe. However, in calculations so far, PBOs near $1200 \mathrm{~Hz}$ occur at $0.4 L_{\mathrm{Edd}}$, a factor of 2 lower than the inferred luminosity when we observed $1200 \mathrm{~Hz}$ QPOs. Calculations for luminosities between $0.4 L_{\mathrm{Edd}}$ and $1.1 L_{\mathrm{Edd}}$ covering a wider range of parameters are needed to compare the properties of the PBOs more accurately with the observed properties of the $\mathrm{kHz}$ QPOs in $Z$ sources.

GX $17+2$ and Sco X-1 have similar timing properties. The highest observed frequency of the $\mathrm{kHz}$ QPOs in both sources is approximately $1100 \mathrm{~Hz}$, and the peak separations are more or less equal (see Fig. 4). However, several aspects of the timing behavior differ significantly between the two sources. In Sco $\mathrm{X}-1$ the highest frequency for the $\mathrm{kHz}$ QPOs is observed at the beginning of the FB (van der Klis et al. 1996a), but in GX $17+2$ this frequency is already reached on the upper NB. This difference is remarkable, because both sources are thought to reach $L_{\mathrm{Edd}}$ near the NB/FB vertex (i.e., $S_{z}=2$ ). So the same frequency is reached in GX 17+2 farther from the Eddington critical value than in Sco X-1.

Also, in Sco X-1 the frequency of the HBO does not decrease as $M$ increases (van der Klis et al. 1997b). Wijnands et al. (1996) suggested that an increase in the mass flux through the radial inflow as GX $17+2$ moves down the NB could produce the decrease of mass flux through the inner disk needed in the magnetospheric BFM to explain the observed decrease of the HBO frequency with increasing $M$. It remains to be seen if indications can be found for a different radial inflow behavior between the two sources, as would be required to explain the difference in the HBO frequency changes with $\dot{M}$ in this framework.

This work was supported in part by the Netherlands Foundation for Research in Astronomy (ASTRON) grant 781-76017 and by NSF grant AST 93-15133. B. V. (NAG 5-3340), F. K. L. (NAG 5-2925), J. v. P. (NAG 5-3269, NAG 5-3271), and W. H. G. L. acknowledge support from NASA grants. M. $\mathrm{M}$. is a fellow of the Consejo Nacional de Investigaciones Científicas y Técnicas de la República Argentina.

\section{REFERENCES}

Alpar, M. A., \& Shaham, J. 1985, Nature, 316, 239

Hasinger, G., \& van der Klis, M. 1989, A\&A, 225, 79

Hasinger, G., van der Klis, M., Ebisawa, K., Dotani, T., \& Mitsuda, K. 1990, A\&A, 235, 131

Homan, J., et al. 1997, in preparation

Klein, R. L., Jernigan, G. J., Arons, J., Morgan, E. H., \& Zhang, W. 1996, ApJ, 469, L119

Kuulkers, E., van der Klis, M., Oosterbroek, T., Asai, K., Dotani, T., van Paradijs, J., \& Lewin, W. H. G. 1994, A\&A, 289, 795

Kuulkers, E., van der Klis, M., Oosterbroek, T., van Paradijs, J., \& Lewin, W. H. G. 1997, MNRAS, 287, 495

Lamb, F. K., Shibazaki, N., Alpar, M. A., \& Shaham, J. 1985, Nature, 317, 681

Miller, M. C., Lamb, F. K., \& Psaltis, D. 1997, ApJ, submitted

van der Klis, M. 1989, in Timing Neutron Stars, ed. H. Ögelman \& E. P. J. van den Heuvel (Dordrecht: Kluwer), 27

. 1997, in The Many Faces of Neutron Stars (NATO/ASI; Dordrecht: Kluwer), in press (astro-ph/9710016) van der Klis, M., et al. 1997a, IAU Circ. 6565

van der Klis, M., Swank, J. H., Zhang, W., Jahoda, K., Morgan, E. H., Lewin, W. H. G., Vaughan, B., \& van Paradijs, J. 1996a, ApJ, 469, L1

van der Klis, M., Wijnands, R. A. D., Horne, K., \& Chen, W. 1997b, ApJ, 481, L97

van der Klis, M., Wijnands, R. A. D., van Paradijs, J., Lewin, W. H. G., Lamb, F. K., Vaughan, B., Kuulkers, E., Psaltis, D., \& Dieters, S. 1996b, IAU Circ. 6511

Wijnands, R., et al. 1997a, ApJL, submitted

Wijnands, R. A. D., \& van der Klis, M. 1997, ApJ, 482, L65

Wijnands, R. A. D., van der Klis, M., Kuulkers, E., Asai, K., Hasinger, G. 1997b, A\&A, 323, 399

Wijnands, R. A. D., van der Klis, M., Psaltis, D., Lamb, F. K., Kuulkers, E., Dieters, S., van Paradijs, J., \& Lewin, W. H. G. 1996, ApJ, 469, L5

Zhang, W. 1995, XTE/PCA internal memo

Zhang, W., Jahoda, K., Swank, J. H., Morgan, E. H., \& Giles, A. B. 1995, ApJ, 449, 930 